Ramanujan Journal 12 (2006), 439-454

\title{
ANALYSIS OF SOME NEW PARTITION STATISTICS
}

\author{
PETER J. GRABNER ${ }^{\dagger}$ AND ARNOLD KNOPFMACHER ${ }^{\ddagger}$
}

Dedicated to Helmut Prodinger on the occasion of his $50^{\text {th }}$ birthday

\begin{abstract}
We study several statistics for integer partitions: for a random partition of an integer $n$ we consider the average size of the smallest gap (= missing part size), the multiplicity of the largest part, and the largest repeated part size. Furthermore, we estimate the number of gap-free partitions of $n$.
\end{abstract}

\section{INTRODUCTION}

The study of partition statistics can be said to have begun with Erdős and Lehner [3] in 1941, who studied questions concerning the normal, resp. average value over all partitions of $n$ of quantities such as the number of parts, the number of different part sizes, and the size of the largest part.

To begin with, instead of looking at parts in partitions we will look at gaps, that is, at part sizes that do not appear in the partition. A partition of a natural number $n$ is said to be gap-free if the part sizes occurring in it form an interval. This idea was studied for partitions of sets by Goh and Schmutz [4], where it was shown that a majority of partitions of an $n$-element set are gap-free with respect to the sizes of the blocks. By contrast we show that gap-free integer partitions are scarce. In Theorem 2, we show that gap-free partitions are in fact close in number to partitions of $n$ with distinct parts. The asymptotic of partitions with distinct parts was studied by Hua in [5].

As almost all partitions of $n$ do contain gaps, another statistic of interest is the size of the least gap. In Section 4 we derive generating functions for this, as well as asymptotic estimates given in Theorem 3.

We then turn our attention to the largest parts in partitions. As already mentioned, the size of the largest part in a random partition of $n$ has been studied by Erdös and Lehner. In Section 5 we extend their investigation by studying the multiplicity of the largest part in a random partition of $n$. Since this multiplicity turns out to be 1 (see Theorem 4), it is natural to then study the largest repeated part size in a random partition of $n$. In Section 6 we show that the largest repeated part size is on average half the size of the largest part. Theorem 5 provides a more precise estimate.

Date: February 14, 2008.

1991 Mathematics Subject Classification. Primary: 05A17; Secondary: 11P82.

Key words and phrases. Partitions.

$\dagger$ This author is supported by the START-project Y96-MAT of the Austrian Science Fund.

$\ddagger$ This material is based upon work supported by the National Research Foundation under grant number 2053740 . 


\section{GAP-FREE PARTITIONS}

A partition of a natural number $n$ is said to be gap-free if the part sizes occurring in it form an interval. In addition if the interval starts at 1, the partition is said to be complete.

Example. Of the 11 partitions of $n=6$, there are 7 gap-free partitions

$$
6,3+3,3+2+1,2+2+2,2+2+1+1,2+1+1+1+1,1+1+1+1+1+1
$$

and 4 complete partitions

$$
3+2+1,2+2+1+1,2+1+1+1+1,1+1+1+1+1+1 .
$$

The generating function for complete partitions is

$$
1+\sum_{j=1}^{\infty} \prod_{i=1}^{j} \frac{z^{i}}{1-z^{i}}=1+\sum_{j=1}^{\infty} \frac{z^{j(j+1) / 2}}{(1-z)\left(1-z^{2}\right) \ldots\left(1-z^{j}\right)}=\prod_{m=1}^{\infty}\left(1+z^{m}\right) .
$$

The last equality holds by one of Euler's partition identities.

Thus we have the following result.

Theorem 1. The number of complete partitions of $n$ equals the number of distinct partitions of $n$.

Combinatorial Proof. The conjugate of the Ferrers' graph of a complete partition gives a bijection with distinct partitions.

The asymptotic growth of distinct partitions is known. There is an exact and asymptotic formula similar to the famous Hardy-Ramanujan-Rademacher formula, which was given by Hua [5].

Now the generating function for gap-free partitions with largest part $j$ is

$$
\sum_{r=1}^{j} \prod_{i=r}^{j} \frac{z^{i}}{1-z^{i}}
$$

Hence the generating function for gap-free partitions is

$$
1+\sum_{j=1}^{\infty} \sum_{r=1}^{j} \prod_{i=r}^{j} \frac{z^{i}}{1-z^{i}}
$$

In this case if we transpose the Ferrers' graph of a gap-free partition we obtain

Proposition 1. The number of gap-free partitions of $n$ equals the number of partitions of $n$ where the largest part may be repeated and all other parts are distinct.

Using this equivalence we get an alternative simpler generating function for gap-free partitions:

$$
1+\sum_{j=1}^{\infty} \frac{z^{j}}{1-z^{j}} \prod_{i=1}^{j-1}\left(1+z^{i}\right)
$$


The sequence of the number of gap-free partitions of $n$ for $n=1, \ldots, 25$ is

$1,2,3,4,5,7,8,10,13,15,18,23,26,31,39,44,52,63,72,85,101,115,134,158,181$.

This is sequence A034296 in Sloane's Online Encyclopedia of Integer Sequences [9].

We use (2.1) to derive a formula for $g(n)$, the number of gap-free partitions of $n$. Now for fixed $j$,

$$
\frac{z^{j}}{1-z^{j}} \prod_{i=1}^{j-1}\left(1+z^{i}\right)
$$

is the generating function for partitions of $n$ with largest part of size $j$ and smaller parts distinct. Denote by $q(n, j)$ the number of distinct partitions of $n$ with largest part $j$. Then

$$
\begin{aligned}
{\left[z^{n}\right] \frac{z^{j}}{1-z^{j}} \prod_{i=1}^{j-1}\left(1+z^{i}\right)=\left[z^{n}\right] \prod_{i=1}^{j-1}\left(1+z^{i}\right)\left(z^{j}\right.} & \left.+z^{2 j}+z^{3 j}+\ldots\right) \\
& =q(n, j)+q(n-j, j)+q(n-2 j, j)+\ldots
\end{aligned}
$$

Summing over $j$ gives the formula

$$
g(n)=\sum_{j \geq 1} \sum_{k \geq 0} q(n-k j, j)=q(n)+\sum_{j \geq 1} \sum_{k \geq 1} q(n-k j, j) .
$$

The number of gap-free but not complete partitions of $n$ is also of interest. For $n=$ $1, \ldots, 25$ this sequence is

$$
0,1,1,2,2,3,3,4,5,5,6,8,8,9,12,12,14,17,18,21,25,26,30,36,39 \text {. }
$$

This sequence is not in Sloane's Encyclopedia [9].

Conjugation of the Ferrers' graph gives: The number of gap-free but not complete partitions of $n$ equals the number of partitions of $n$ where the largest part occurs at least twice and all other parts are distinct.

The generating function for this sequence is thus

$$
\sum_{j=1}^{\infty} \frac{z^{2 j}}{1-z^{j}} \prod_{i=1}^{j-1}\left(1+z^{i}\right)
$$

\section{Asymptotic Estimates for Gap-Free partitions}

In order to derive the asymptotic behaviour of gap-free partitions, we first investigate the inner sum in (2.2) for small $k$. From [11, Theorem 2] we have that

$$
q(n, j)=q(n) \exp \left(-\frac{\pi j}{2 \sqrt{3} \sqrt{n}}-\frac{2 \sqrt{3}}{\pi} \sqrt{n} \exp \left(-\frac{\pi j}{2 \sqrt{3} \sqrt{n}}\right)+\mathcal{O}\left(n^{-\frac{1}{2}}\right)\right)
$$

uniformly for $\frac{2 \sqrt{3}}{3 \pi} \sqrt{n} \log n+\mathcal{O}(\sqrt{n}) \leq j \leq \frac{4 \sqrt{3}}{3 \pi} \sqrt{n} \log n+\mathcal{O}(\sqrt{n})$ (the error term is not explicitly given in [11], but can be derived by an immediate adaptation of the method used 
there). Inserting

$$
q(n)=\frac{1}{4 \cdot 3^{\frac{1}{4}} n^{\frac{3}{4}}} \exp \left(\frac{\pi}{\sqrt{3}} \sqrt{n}\right)\left(1+\mathcal{O}\left(n^{-\frac{1}{2}}\right)\right)
$$

into (3.1) we obtain for $k \leq 4 \log n$

$$
q(n-k j, j)=q(n) \exp \left(-\frac{\pi(k+1) j}{2 \sqrt{3} \sqrt{n}}-\frac{2 \sqrt{3}}{\pi} \sqrt{n} \exp \left(-\frac{\pi j}{2 \sqrt{3} \sqrt{n}}\right)+\mathcal{O}\left(n^{-\frac{1}{3}} \log ^{3} n\right)\right)
$$

Thus we have for $k \leq 4 \log n$

$$
\begin{array}{r}
\sum_{j \geq 1} q(n-k j, j)=q(n) \sum_{j \in R_{n}} \exp \left(-\frac{\pi(k+1) j}{2 \sqrt{3} \sqrt{n}}-\frac{2 \sqrt{3}}{\pi} \sqrt{n} \exp \left(-\frac{\pi j}{2 \sqrt{3} \sqrt{n}}\right)+\mathcal{O}\left(n^{-\frac{1}{3}} \log ^{3} n\right)\right) \\
+\sum_{\text {rest }} q(n-k j, j),
\end{array}
$$

where $R_{n}=\left\{j \in \mathbb{Z} \mid \frac{2 \sqrt{3}}{3 \pi} \sqrt{n} \log n \leq j \leq \frac{4 \sqrt{3}}{3 \pi} \sqrt{n} \log n\right\}$. Setting $x_{j}=\frac{j}{\sqrt{n}}$ we obtain

$$
\begin{aligned}
& \sum_{j \in R_{n}} q(n-k j, j) \\
= & q(n) \sqrt{n} \sum_{j \in R_{n}} \exp \left(-\frac{\pi}{2 \sqrt{3}}(k+1) x_{j}-\frac{2 \sqrt{3}}{\pi} \exp \left(-\frac{\pi}{2 \sqrt{3}} x_{j}+\frac{1}{2} \log n\right)\right) \frac{1}{\sqrt{n}}\left(1+\mathcal{O}\left(n^{-\frac{1}{3}} \log ^{3} n\right)\right) .
\end{aligned}
$$

This sum is now a Riemann sum for the integral

$$
\begin{gathered}
\int_{\frac{2 \sqrt{3}}{3 \pi} \log n}^{\frac{4 \sqrt{3}}{3 \pi} \log n} \exp \left(-\frac{\pi}{2 \sqrt{3}}(k+1) x-\frac{2 \sqrt{3}}{\pi} \exp \left(-\frac{\pi}{2 \sqrt{3}} x+\frac{1}{2} \log n\right)\right) d x= \\
n^{-\frac{k+1}{2}}\left(\frac{\pi}{2 \sqrt{3}}\right)^{k} \int_{\frac{2 \sqrt{3}}{\pi} n^{-\frac{1}{6}}}^{\frac{2 \sqrt{3}}{\pi} n^{\frac{1}{6}}} y^{k} e^{-y} d y=k ! n^{-\frac{k+1}{2}}\left(\frac{\pi}{2 \sqrt{3}}\right)^{k}\left(1+\mathcal{O}\left(n^{-\frac{k+1}{6}}\right)\right) .
\end{gathered}
$$

The first derivative of the integrand is bounded by $\mathcal{O}\left(\left(\frac{\pi}{2 \sqrt{3 n}}\right)^{k+1} k^{\frac{3}{2}} k !\right)$. Thus the difference between the integral and the Riemann sum is bounded by $\mathcal{O}\left(\left(\frac{\pi}{2 \sqrt{3 n}}\right)^{k+1} k^{\frac{3}{2}} k ! n^{-\frac{1}{2}} \log n\right)$. 
The remaining sum in (3.3) is split into two parts which are estimated as follows:

$$
\begin{aligned}
\sum_{j<\frac{2 \sqrt{3}}{3 \pi} \sqrt{n} \log n} q(n-k j, j)=\mathcal{O}( & \left.\sqrt{n}(\log n) n^{\frac{1}{3}} q\left(n,\left[\frac{2 \sqrt{3}}{3 \pi} \sqrt{n} \log n\right]+1\right)\right)= \\
& \mathcal{O}\left(\sqrt{n}(\log n) n^{\frac{1}{3}} q(n) \exp \left(-\frac{2 \sqrt{3}}{\pi} n^{\frac{1}{6}}-\frac{1}{3} \log n\right)\right)
\end{aligned}
$$

using (3.1);

$$
\begin{aligned}
\sum_{j>\frac{4 \sqrt{3}}{3 \pi} \sqrt{n} \log n} q(n-k j, j) \leq \sum_{j>\frac{4 \sqrt{3}}{3 \pi} \sqrt{n} \log n} q(n-(k+1) j)= \\
\mathcal{O}\left(q(n) \sum_{j>\frac{4 \sqrt{3}}{3 \pi} \sqrt{n} \log n} \exp \left(-\frac{\pi}{\sqrt{3}} \frac{(k+1) j}{2 \sqrt{n}}\right)\right)=\mathcal{O}\left(q(n) n^{-k / 2-(k+1) / 6}\right)
\end{aligned}
$$

by using (3.2).

Putting everything together we obtain for $k \leq 4 \log n$

$$
\sum_{j \geq 1} q(n-k j, j)=q(n) n^{-\frac{k}{2}} k !\left(\frac{\pi}{2 \sqrt{3}}\right)^{k}\left(1+\mathcal{O}\left(n^{-\frac{1}{3}} \log ^{3} n\right)\right) .
$$

We now split the range of summation with respect to $k$ into four parts

$$
\sum_{j} q(n-j, j)+\sum_{2 \leq k \leq 4 \log n} \sum_{j} q(n-k j, j)+\sum_{4 \log n<k \leq 6 \frac{n}{\log n}} \sum_{j} q(n-k j, j)+\sum_{k>6 \frac{n}{\log n}} \sum_{j} q(n-k j, j) .
$$

The first sum equals $\frac{\pi}{2 \sqrt{3}} q(n) n^{-\frac{1}{2}}\left(1+\mathcal{O}\left(n^{-\frac{1}{3}} \log ^{3} n\right)\right)$ by (3.6). The second sum is estimated by $\mathcal{O}\left(q(n) n^{-1}\right)$ again using (3.6). For the third summand we observe that

$$
q(n-k j, j) \neq 0 \quad \text { if and only if }(k+1) j \leq n \leq \frac{j(j+2 k+1)}{2} .
$$

The second inequality is satisfied only if $j \geq \frac{4 \sqrt{n} \log n}{k}$ (for $k \geq 4 \log n$ and large enough $n$ ). The inner sum in the third sum has $\mathcal{O}(n / k)$ terms. Furthermore,

$$
q(n-k j, j) \leq q(n-k j) \leq q(n-[4 \sqrt{n} \log n])=\mathcal{O}\left(q(n) n^{-3}\right) .
$$

Thus we can estimate the third sum by

$$
\mathcal{O}\left(q(n) n^{-3} \sum_{4 \log n \leq k \leq 6 \frac{n}{\log n}} \frac{n}{k}\right)=\mathcal{O}\left(q(n) n^{-2} \log n\right) .
$$

In the fourth sum we have $\mathcal{O}(\log n)$ summands in the inner sum; each of these summands can be estimated by $q(n) n^{-2}$.

Putting everything together we have proved 
Theorem 2. The number $g(n)$ of gap-free partitions of $n$ satisfies

$$
g(n)=q(n)+\frac{\pi}{2 \sqrt{3}} \frac{q(n)}{\sqrt{n}}+\mathcal{O}\left(q(n) n^{-5 / 6} \log ^{3} n\right) .
$$

\section{Smallest Gaps in Partitions}

We consider all partitions of the number $n$ and investigate the average size of the smallest gap. That is, the least part size that is not present in the partition.

Example. $1+3+7$ has least gap $2 ; 2+5$ has least gap 1 and $1+2+3$ has least gap 4 .

Let $r(n, k)$ be the number of partitions of $n$ with least gap greater than $k$. Then

$$
\begin{aligned}
& r_{k}(z):=\sum_{n=0}^{\infty} r(n, k) z^{n}=\prod_{i=1}^{k} \frac{z^{i}}{1-z^{i}} \prod_{i=k+1}^{\infty} \frac{1}{1-z^{i}}=z^{\frac{k(k+1)}{2}} \prod_{i=1}^{\infty} \frac{1}{1-z^{i}} \\
&=z^{\frac{k(k+1)}{2}} \sum_{j=0}^{\infty} p(j) z^{j},
\end{aligned}
$$

where $P(z):=\sum_{j=0}^{\infty} p(j) z^{j}$ is the generating function for the number of partitions of $n$.

Define $\ell(n)$ to be the sum of the least gaps over all partitions of $n$. Then

$$
L(z):=\sum_{n=0}^{\infty} \ell(n) z^{n}=\sum_{k=0}^{\infty} r_{k}(z)=\sum_{k=0}^{\infty} z^{\frac{k(k+1)}{2}} \sum_{j=0}^{\infty} p(j) z^{j} .
$$

From the identity (cf. [1, (10.4.8)])

$$
\sum_{k=0}^{\infty} z^{\frac{k(k+1)}{2}}=\prod_{n=1}^{\infty} \frac{1-z^{2 n}}{1-z^{2 n-1}}
$$

we derive

$$
L(z)=\prod_{n=1}^{\infty}\left(1+z^{n}\right)^{2}=Q(z)^{2},
$$

where $Q(z)$ denotes the generating function of distinct partitions.

The transformation formulæ for $Q(z)=\frac{P(z)}{P\left(z^{2}\right)}$ are given in [5] or can be derived from the transformation rules of $P(z)$ : for $(h, k)=1, h h^{\prime} \equiv-1(\bmod k), 2 h h^{\prime \prime} \equiv-1(\bmod k)$ (for $k$ odd), and $h h^{\prime \prime \prime} \equiv-1\left(\bmod \frac{k}{2}\right)$ (for $k$ even) we have

$$
\begin{aligned}
Q\left(\exp \left(2 \pi i \frac{h}{k}-\frac{2 \pi z}{k^{2}}\right)\right)= \\
\begin{cases}\exp \left(\pi i\left(s(h, k)-s\left(h, \frac{k}{2}\right)\right)\right) \exp \left(-\frac{\pi}{12 z}+\frac{\pi z}{12 k^{2}}\right) \frac{P\left(\exp \left(2 \pi i \frac{h^{\prime}}{k}-\frac{2 \pi}{z}\right)\right)}{P\left(\exp \left(\pi i \frac{h^{\prime \prime \prime}}{k}-\frac{4 \pi}{z}\right)\right)} & \text { for } k \text { even } \\
\frac{1}{\sqrt{2}} \exp (\pi i(s(h, k)-s(2 h, k))) \exp \left(\frac{\pi}{24 z}+\frac{\pi z}{12 k^{2}}\right) \frac{P\left(\exp \left(2 \pi i \frac{h^{\prime}}{k}-\frac{2 \pi}{z}\right)\right)}{P\left(\exp \left(2 \pi i \frac{h^{\prime \prime}}{k}-\frac{\pi}{z}\right)\right)} & \text { for } k \text { odd, }\end{cases}
\end{aligned}
$$


from which we derive that

$$
\begin{aligned}
& L\left(\exp \left(2 \pi i \frac{h}{k}-\frac{2 \pi z}{k^{2}}\right)\right)= \\
& \qquad \begin{cases}\exp \left(2 \pi i\left(s(h, k)-s\left(h, \frac{k}{2}\right)\right)\right) \exp \left(-\frac{\pi}{3 z}+\frac{\pi z}{6 k^{2}}\right)\left(1+\mathcal{O}\left(\exp \left(-\frac{4 \pi}{z}\right)\right)\right) & \text { for } k \text { even } \\
\frac{1}{2} \exp (2 \pi i(s(h, k)-s(2 h, k))) \exp \left(\frac{\pi}{12 z}+\frac{\pi z}{6 k^{2}}\right)\left(1+\mathcal{O}\left(\exp \left(-\frac{\pi}{z}\right)\right)\right) & \text { for } k \text { odd },\end{cases}
\end{aligned}
$$

where the implied constants are absolute. Now $\ell(n)$ can be written as

$$
\ell(n)=\sum_{\substack{0 \leq h<k \leq N \\(h, k)=1}} \frac{i}{k^{2}} e^{-2 \pi i \frac{h n}{k}} \int_{z_{h k}^{\prime}}^{z_{h k}^{\prime \prime}} L\left(\exp \left(2 \pi i \frac{h}{k}-\frac{2 \pi z}{k^{2}}\right)\right) \exp \left(\frac{2 \pi n z}{k^{2}}\right) d z
$$

as in $\left[8\right.$, p. 268], where $z$ runs on the circle $\left|z-\frac{1}{2}\right|=\frac{1}{2}$ on the arc between

$$
z_{h k}^{\prime}=\frac{k^{2}}{k^{2}+k_{1}^{2}}+i \frac{k k_{1}}{k^{2}+k_{1}^{2}} \text { and } z_{h k}^{\prime \prime}=\frac{k^{2}}{k^{2}+k_{2}^{2}}-i \frac{k k_{2}}{k^{2}+k_{2}^{2}} .
$$

Here $\frac{h_{1}}{k_{1}}, \frac{h}{k}$, and $\frac{h_{2}}{k_{2}}$ are three adjacent Farey fractions with denominators $\leq N$.

Now (4.3) and the argument given in [5, section 6] show that the terms in (4.4) with $k$ even contribute only $\mathcal{O}\left(N^{-\frac{1}{3}+\varepsilon}\right)$. The terms with $k$ odd are treated in the same way as in $[8$, pp. 270] or [5, section 7]. This yields a contribution

$$
\int_{\left|z-\frac{1}{2}\right|=\frac{1}{2}} \exp \left(\frac{\pi}{12 z}+\frac{2 \pi z}{k^{2}}\left(n+\frac{1}{12}\right)\right) d z=\frac{\pi k}{i \sqrt{6} \sqrt{n+\frac{1}{12}}} I_{1}\left(\frac{\pi}{k} \sqrt{\frac{2}{3}} \sqrt{n+\frac{1}{12}}\right)
$$

for all terms with $k$ odd and $(h, k)=1$. Here $I_{1}$ denotes the modified Bessel function of index 1.

Summing up we obtain the asymptotic expansion

$$
\ell(n)=\frac{\pi}{2 \sqrt{6} \sqrt{n+\frac{1}{12}}} \sum_{k=1}^{\infty} \frac{A_{2 k-1}(n)}{(2 k-1)} I_{1}\left(\pi \sqrt{\frac{2}{3}} \frac{\sqrt{n+\frac{1}{12}}}{2 k-1}\right),
$$

where

$$
A_{k}(n)=\sum_{\substack{0 \leq h<k \\(h, k)=1}} \exp \left(2 \pi i\left(s(h, k)-s(2 h, k)-\frac{h n}{k}\right)\right),
$$

where as usual in this context $s(h, k)$ denotes Dedekind's sum

$$
s(h, k)=\sum_{r=1}^{k-1} \frac{r}{k}\left(\frac{h r}{k}-\left\lfloor\frac{h r}{k}\right\rfloor-\frac{1}{2}\right) .
$$


Using the arguments given in [5] the coefficients $A_{k}(n)$ can be identified as finite sums of Kloosterman sums. Weil's bound for these sums gives the estimate

$$
A_{k}(n)=\mathcal{O}\left(k^{\frac{1}{2}} \sigma_{0}(k)\right),
$$

where $\sigma_{0}(k)$ denotes the number of divisors of $k$ (cf. $[6,8]$ ). Combining this with the estimate $I_{1}(x)=\mathcal{O}(x)$ for $x \rightarrow 0$, this gives the bound

$$
\frac{A_{2 k-1}(n)}{(2 k-1)} I_{1}\left(\pi \sqrt{\frac{2}{3}} \frac{\sqrt{n+\frac{1}{12}}}{2 k-1}\right)=\mathcal{O}\left(k^{-\frac{3}{2}} \sigma_{0}(k) n^{\frac{1}{2}}\right)
$$

for $k \geq n^{\frac{1}{2}}$. This implies that the series in (4.5) is indeed convergent.

Using only the first term of (4.5) and the asymptotic expansion (cf. [1])

$$
I_{1}(x) \sim \frac{e^{x}}{\sqrt{2 \pi x}}
$$

yields

$$
\ell(n) \sim \frac{1}{4 \sqrt[4]{6}} n^{-\frac{3}{4}} \exp \left(\pi \sqrt{\frac{2 n}{3}}\right)
$$

Thus we have proved

Theorem 3. The sum $\ell(n)$ of the smallest gaps in all partitions of $n$ satisfies

$$
\sum_{n=0}^{\infty} \ell(n) z^{n}=\prod_{k=1}^{\infty}\left(1+z^{n}\right)^{2} .
$$

The quantity $\ell(n)$ satisfies the exact and asymptotic formula

$$
\ell(n)=\frac{\pi}{2 \sqrt{6} \sqrt{n+\frac{1}{12}}} \sum_{k=1}^{\infty} \frac{A_{2 k-1}(n)}{(2 k-1)} I_{1}\left(\pi \sqrt{\frac{2}{3}} \frac{\sqrt{n+\frac{1}{12}}}{2 k-1}\right),
$$

where $A_{k}(n)$ is given by (4.6). The average size of the smallest gap is then given by

$$
\bar{\ell}(n)=\frac{\ell(n)}{p(n)} \sim \sqrt[4]{\frac{3}{2}} n^{\frac{1}{4}} .
$$

Remark. As the referee pointed out to us, there is a proof similar to the proof of Theorem 2 for (4.7) and for the asymptotic equivalent of $\bar{\ell}(n)$.

The sequence $\ell(n)$ for $n=0, \ldots 25$ is

1, $2,3,6,9,14,22,32,46,66,93,128,176,238,319,426,562,736$,

960, 1242, 1598, 2048, 2608, 3306, 4175, 5248 .

This sequence occurs in Sloane's Encyclopedia [9] as A022567. The definition used there is $(4.8)$. 


\section{Multiplicity of The LARGEST PART}

Here we study the multiplicity of the largest part of a partition.

Example. The partition $1+3+4+6+6$ has multiplicity 2 for its largest part.

By transposing the Ferrers' diagram of a partition with a unique largest part we see immediately that such a partition corresponds to a partition which has a part of size one. Thus the number of partitions of $n$ with a unique largest part is equal to $p(n-1)$.

More generally, transposing the Ferrers' diagram of a partition with largest part of multiplicity $k$ gives a correspondence to a partition which has its least part of size $k$. The generating function for partitions with least part of size $k$ is

$$
z^{k} \prod_{j=0}^{\infty} \frac{1}{\left(1-z^{k+j}\right)}
$$

whilst the generating function for partitions with least part of size $\geq k$ is

$$
-1+\prod_{j=0}^{\infty} \frac{1}{\left(1-z^{k+j}\right)}
$$

Thus the generating function for the total multiplicity of the largest part, summed over all partitions of $n$ is

$$
\sum_{k=1}^{\infty} k z^{k} \prod_{j=0}^{\infty} \frac{1}{\left(1-z^{k+j}\right)}=\sum_{k=1}^{\infty}\left(-1+\prod_{j=0}^{\infty} \frac{1}{\left(1-z^{k+j}\right)}\right) .
$$

The sequence of integers corresponding to this generating function begins 1, 3, 5, 9, 12, 20, 25, 38, 49, 69, 87, 123, 152, 205, 260, 341, 425, 555, 687, 882, 1094, 1380, 1702.

This sequence appears in Sloane's Encyclopedia [9] as A046746 under its alternative interpretation (via the Ferrers' diagram), as the sum of the smallest parts in all partitions of $n$.

The generating function

$$
\frac{1}{1-u z^{k}} \prod_{\ell=1}^{k-1} \frac{1}{1-z^{\ell}}
$$

counts the partitions of $n$ into parts $\leq k$ and the multiplicity of the part $k$ is counted by the exponent of $u$. Thus the total multiplicity of the largest parts has the generating function

$$
\left.\sum_{k=1}^{\infty} \frac{\partial}{\partial u} \frac{1}{1-u z^{k}} \prod_{\ell=1}^{k-1} \frac{1}{1-z^{\ell}}\right|_{u=1}=\sum_{k=1}^{\infty} \frac{z^{k}}{1-z^{k}} \prod_{\ell=1}^{k} \frac{1}{1-z^{\ell}} .
$$

Thus we have proved the identity

$$
\sum_{n=0}^{\infty} t(n) z^{n}=\sum_{k=1}^{\infty} k z^{k} \prod_{j=0}^{\infty} \frac{1}{\left(1-z^{k+j}\right)}=\sum_{k=1}^{\infty} \frac{z^{k}}{1-z^{k}} \prod_{\ell=1}^{k} \frac{1}{1-z^{\ell}}
$$


From this we read off the formula for the total multiplicity $t(n)$ of largest parts in all partitions of $n$

$$
t(n)=\sum_{k=1}^{\infty} \sum_{m=1}^{\infty} p_{k}(n-m k)
$$

where $p_{k}(n)$ denotes the number of partitions of $n$ in parts $\leq k$. We notice that this formula is similar to $(2.2)$.

In order to obtain the asymptotic behaviour of $t(n)$, we treat the sums

$$
\sum_{k=1}^{\infty} p_{k}(n-m k)
$$

for fixed $m \geq 1$. From [3] and [10] we have

$$
\frac{p_{k}(n-m k)}{p(n-m k)}=\exp \left(-\frac{2}{C} \exp \left(-\frac{C}{2} x\right)\right)\left(1+\mathcal{O}\left(\frac{\log ^{2} n}{\sqrt{n}}\right)\right)
$$

for $k=\frac{1}{C} \sqrt{n} \log n+x \sqrt{n}$ and $|x|=o(\log n), C=\pi \sqrt{\frac{2}{3}}$. Furthermore, from the asymptotic expansion of $p(n)$ we get

$$
\frac{p(n-m k)}{p(n)}=\exp \left(-\frac{C m k}{2 \sqrt{n}}\right)\left(1+\mathcal{O}\left(\frac{\log ^{2} n}{\sqrt{n}}\right)\right)=n^{-\frac{m}{2}} \exp \left(-\frac{C x m}{2}\right)\left(1+\mathcal{O}\left(\frac{\log ^{2} n}{\sqrt{n}}\right)\right) \text {. }
$$

Putting (5.2) and (5.3) together we obtain

$$
\frac{p_{k}(n-m k)}{p(n)}=n^{-\frac{m}{2}} \exp \left(-\frac{C x m}{2}-\frac{2}{C} \exp \left(-\frac{C}{2} x\right)\right)\left(1+\mathcal{O}\left(\frac{\log ^{2} n}{\sqrt{n}}\right)\right)
$$

for $k$ in the above mentioned range. Similar arguments as in the proof of Theorem 2 can be applied to compare the asymptotic main term with the integral

$$
\int_{-\infty}^{\infty} \exp \left(-\frac{C x}{2}-\frac{2}{C} \exp \left(-\frac{C}{2} x\right)\right) d x=1
$$

Thus we have

Theorem 4. The average multiplicity of the largest part in a partition of $n$ equals 1 as $n \rightarrow \infty$.

Remark. Theorem 4 is also an immediate consequence of [2, Theorem 1].

\section{LARGEST REPEATED PART SIZE}

We study the largest repeated part size in a partition of $n$. In the case of the partitions with distinct parts we set the largest repeated part size to be 0 .

Example. The partition $1+2+2+2+4+4+5+6$ has largest repeated part size equal to 4 . 
The generating function that counts partitions with largest repeated part equal to $k$ is

$$
\prod_{i=1}^{k-1} \frac{1}{1-z^{i}} \frac{z^{2 k}}{1-z^{k}} \prod_{m=k+1}^{\infty}\left(1+z^{m}\right) .
$$

Combining the products together and summing over $k$ gives the generating function for the sum of the largest repeated part sizes over all partitions of $n$ as

$$
\prod_{i=1}^{\infty} \frac{1}{1-z^{i}} \sum_{k=1}^{\infty} k z^{2 k} \prod_{m=k+1}^{\infty}\left(1-z^{2 m}\right)
$$

We now show that

$$
\sum_{k=1}^{\infty} k z^{k} \prod_{m=k+1}^{\infty}\left(1-z^{m}\right)=\sum_{k=1}^{\infty} \frac{z^{k}}{1-z^{k}}
$$

First multiply the left hand side of (6.2) by

$$
\prod_{n=1}^{\infty} \frac{1}{1-z^{n}}
$$

This gives

$$
\sum_{k=1}^{\infty} k z^{k} \prod_{l=1}^{k} \frac{1}{1-z^{l}}=\sum_{k=1}^{\infty} k \sum_{n \geq 0} p(k, n) z^{n}=\sum_{n \geq 0} z^{n} \sum_{k=1}^{n} k p(k, n),
$$

where $p(k, n)$ denotes the number of partitions of $n$ into exactly $k$ parts.

Now by differentiating the bivariate generating function

$$
\sum_{n, k \geq 0} p(k, n) u^{k} z^{n}=\prod_{n=1}^{\infty} \frac{1}{1-u z^{n}}
$$

with respect to $u$ and then setting $u=1$ we find that

$$
\sum_{n \geq 0} z^{n} \sum_{k=1}^{n} k p(k, n)=\prod_{n=1}^{\infty} \frac{1}{1-z^{n}} \sum_{k=1}^{\infty} \frac{z^{k}}{1-z^{k}}
$$

which proves $(6.2)$.

From (6.1) and (6.2) we thus obtain the generating function for the sum of the largest repeated part sizes over all partitions of $n$ in the simpler form

$$
\prod_{i=1}^{\infty} \frac{1}{1-z^{i}} \sum_{k=1}^{\infty} \frac{z^{2 k}}{1-z^{2 k}} .
$$

The sequence $\{f(n)\}$ enumerating the sum of the largest repeated part sizes over all partitions of $n$ begins

$0,1,1,4,5,11,15,28,38,62,85,131,177,258,346,489,648,890,1168,1572,2042,2699$. 
This is sequence $A 066898$ in [9] where it appears under the heading, total number of even parts in all partitions of $n$.

Equating coefficients of $z^{n}$ gives

$$
f(n)=\sum_{k=1}^{\left\lfloor\frac{n}{2}\right\rfloor} \sigma_{0}(k) p(n-2 k)
$$

where again $\sigma_{0}(k)$ denotes the number of divisors of $k$. This formula is also given in Sloane $[9]$.

Theorem 5. The average value $\bar{f}(n)$ of the largest repeated part in an integer partition of $n$ equals

$$
\bar{f}(n)=\frac{\sqrt{6}}{4 \pi} \sqrt{n} \log n+\sqrt{n}\left(\frac{1}{\pi} \sqrt{\frac{3}{2}} \gamma+\frac{\sqrt{6}}{4 \pi} \log \frac{3}{2 \pi^{2}}\right)+\mathcal{O}(\log n) .
$$

Proof. We study the asymptotic behaviour of the function $f(n)$ given by (6.5). Since

$$
p(n)=\frac{1}{4 n \sqrt{3}} \exp \left(\pi \sqrt{\frac{2}{3} n}\right)\left(1+\mathcal{O}\left(n^{-\frac{1}{2}}\right)\right)
$$

we have for $2 k \leq n^{5 / 8}$,

$$
\frac{p(n-2 k)}{p(n)}=\exp \left(-\pi \sqrt{\frac{2}{3}} \frac{k}{\sqrt{n}}\right)\left(1+\mathcal{O}\left(\frac{k}{n}\right)+\mathcal{O}\left(\frac{k^{2}}{n^{\frac{3}{2}}}\right)+\mathcal{O}\left(\frac{1}{\sqrt{n}}\right)\right) .
$$

Thus we have for $\frac{1}{2}<\alpha<\frac{5}{8}$

$$
\begin{aligned}
f(n)=p(n) \sum_{k \leq n^{\alpha}} \sigma_{0}(k) \exp \left(-\pi \sqrt{\frac{2}{3}} \frac{k}{\sqrt{n}}\right)\left(1+\mathcal{O}\left(\frac{k}{n}\right)\right. & \left.+\mathcal{O}\left(\frac{k^{2}}{n^{\frac{3}{2}}}\right)+\mathcal{O}\left(\frac{1}{\sqrt{n}}\right)\right) \\
& +\sum_{n^{\alpha}<k \leq \frac{n}{2}} \sigma_{0}(k) p(n-2 k) .
\end{aligned}
$$

The second sum can be estimated by

$$
\sum_{n^{\alpha}<k \leq \frac{n}{2}} \sigma_{0}(k) p(n-2 k) \leq p\left(n-2 n^{\alpha}\right) \sum_{k<\frac{n}{2}} \sigma_{0}(k)=\mathcal{O}\left(p(n) \exp \left(-\pi \sqrt{\frac{2}{3}} n^{\alpha-\frac{1}{2}}\right) n \log n\right) .
$$

In order to study the first sum, we introduce the function

$$
g(t)=\sum_{k=1}^{\infty} \sigma_{0}(k) \exp (-k t) .
$$


Then the first sum in $(6.7)$ is almost $g\left(\pi \sqrt{\frac{2}{3 n}}\right)$ : we have

$$
\begin{array}{r}
g\left(\pi \sqrt{\frac{2}{3 n}}\right)-\sum_{k \leq n^{\alpha}} \sigma_{0}(k) \exp \left(-\pi \sqrt{\frac{2}{3}} \frac{k}{\sqrt{n}}\right)=\sum_{k>n^{\alpha}} \sigma_{0}(k) \exp \left(-\pi \sqrt{\frac{2}{3}} \frac{k}{\sqrt{n}}\right) \leq \\
\sum_{k>n^{\alpha}} k \exp \left(-\pi \sqrt{\frac{2}{3}} \frac{k}{\sqrt{n}}\right)=\mathcal{O}\left(n^{1+\alpha} \exp \left(-\pi \sqrt{\frac{2}{3}} n^{\alpha-\frac{1}{2}}\right)\right) .
\end{array}
$$

The asymptotic behaviour of $g(t)$ for $t \rightarrow 0$ can be derived using Mellin transform. By Mellin's inversion formula we have

$$
\begin{aligned}
& g(t)=\frac{1}{2 \pi i} \int_{2-i \infty}^{2+i \infty} \zeta(s)^{2} \Gamma(s) t^{-s} d s= \\
& t^{-1} \log t^{-1}+\gamma t^{-1}+\frac{1}{4}+\frac{1}{2 \pi i} \int_{-\frac{1}{2}-i \infty}^{-\frac{1}{2}+i \infty} \zeta(s)^{2} \Gamma(s) t^{-s} d s=t^{-1} \log t^{-1}+\gamma t^{-1}+\frac{1}{4}+\mathcal{O}\left(t^{\frac{1}{2}}\right) .
\end{aligned}
$$

The contribution, which comes from the two $\mathcal{O}$-terms in the first sum of (6.7) can be estimated in the same way as $g(t)$. This yields a contribution of $\mathcal{O}(\log n)$. Thus we have

$$
\bar{f}(n)=\frac{f(n)}{p(n)}=g\left(\pi \sqrt{\frac{2}{3 n}}\right)+\mathcal{O}(\log n),
$$

from which we obtain the desired result by inserting (6.9).

Remark. In principle, it would be possible to derive a full asymptotic series for $\bar{f}(n)$ involving decreasing powers of $n$ and logarithmic terms.

Acknowledgement. The authors are indebted to an anonymous referee for pointing out a number of errors and making several suggestions for improving the style of the presentation.

\section{REFERENCES}

[1] G. E. Andrews, R. Askey, and R. Roy, Special functions, Encyclopedia of Mathematics and its Applications, vol. 71, Cambridge University Press, Cambridge, 1999.

[2] J. Dixmier and J.-L. Nicolas, Partitions without small parts, Colloq. Math. Soc. János Bolyai 51 (1987), 9-33, North-Holland, 1990.

[3] P. Erdös and J. Lehner, The distribution of the number of summands in the partitions of a positive integer, Duke Math. J. 8 (1941), 335-345.

[4] W. M. Y. Goh and E. Schmutz, Gap-free set partitions, Random Structures Algorithms 3 (1992), 9-18.

[5] L.-K. Hua, On the number of partitions of a number into unequal parts, Trans. Amer. Math. Soc. 51 (1942), 194-201.

[6] H. Iwaniec and E. Kowalski, Analytic number theory, American Mathematical Society Colloquium Publications, vol. 53, American Mathematical Society, Providence, RI, 2004.

[7] H. Rademacher, On the expansion of the partition function in a series, Ann. of Math. (2) 44 (1943), 416-422. 
[8] _ Topics in Analytic Number Theory, Grundlehren der mathematischen Wissenschaften, vol. 169, Springer-Verlag, New York, 1973, Edited by E. Grosswald, J. Lehner and M. Newman.

[9] N. J. A. Sloane and S. Plouffe, The encyclopedia of integer sequences, Academic Press Inc., San Diego, CA, 1995, Online edition available at http://www.research.att.com/ njas/sequences/.

[10] G. Szekeres, Some asymptotic formulae in the theory of partitions. II, Quart. J. Math., Oxford Ser. (2) 4 (1953), 96-111.

[11] Asymptotic distribution of the number and size of parts in unequal partitions, Bull. Austral. Math. Soc. 36 (1987), 89-97.

Peter Grabner, Institut für Mathematik A, Technische Universität Graz, Steyrergasse 30, 8010 Graz, Austria

E-mail address: peter.grabner@tugraz.at

Arnold Knopfmacher, The John Knopfmacher Centre for Applicable Analysis and Number Theory, Department of Mathematics, University of the Witwatersrand, P. O. Wits, 2050 Johannesburg, South Africa

E-mail address: arnoldkn@cam.wits.ac.za

URL: http://www.wits.ac.za/science/number_theory/arnold.htm 